\title{
Hypergolic Materials Synthesis through Reaction of Fuming Nitric Acid with Certain Cyclopentadienyl Compounds
}

\author{
Nikolaos Chalmpes ${ }^{1}$, , Athanasios B. Bourlinos ${ }^{2, *}$, Veronika Šedajová ${ }^{3}$ (D) , Vojtěch Kupka $^{3}$, \\ Dimitrios Moschovas ${ }^{1}$, Apostolos Avgeropoulos ${ }^{1} \mathbb{D}$, Michael A. Karakassides ${ }^{1}$ \\ and Dimitrios Gournis $1, *$ (D) \\ 1 Department of Materials Science \& Engineering, University of Ioannina, 45110 Ioannina, Greece; \\ chalmpesnikos@gmail.com (N.C.); dmoschov@cc.uoi.gr (D.M.); aavger@uoi.gr (A.A.); \\ mkarakas@uoi.gr (M.A.K.) \\ 2 Physics Department, University of Ioannina, 45110 Ioannina, Greece \\ 3 Regional Centre of Advanced Technologies and Materials, Faculty of Science, Palacky University in \\ Olomouc, Slechtitelu 27, 77900 Olomouc, Czech Republic; veronika.sedajova@upol.cz (V.Š.); \\ vojtech.kupka@upol.cz (V.K.) \\ * Correspondence: bourlino@uoi.gr (A.B.B.); dgourni@uoi.gr (D.G.); Tel.: +30-26510-07141 (D.G.)
}

Received: 17 August 2020; Accepted: 2 October 2020; Published: 5 October 2020

\begin{abstract}
Recently we have shown the importance of hypergolic reactions in carbon materials synthesis. However, hypergolic reactions could be certainly expanded beyond carbon synthesis, offering a general preparative pathway towards a larger variety of materials. Cyclopentadienyls are one of the most common ligands in organometallic chemistry that react hypergolicly on contact with strong oxidizers. By also considering the plethora of cyclopentadienyl compounds existing today, herein we demonstrate the potential of such compounds in hypergolic materials synthesis in general (carbon or inorganic). In a first example, we show that cyclopentadienyllithium reacts hypergolicly with fuming nitric acid to produce carbon. In a second one, we show that ferrocene and cobaltocene also react hypergolicly with the concentrated acid to afford magnetic inorganic materials, such as $\gamma-\mathrm{Fe}_{2} \mathrm{O}_{3}$ and metallic $\mathrm{Co}$, respectively. The present results further emphasize the importance and universal character of hypergolic reactions in materials science synthesis, as an interesting new alternative to other existing and well-established preparative methods.
\end{abstract}

Keywords: materials synthesis; hypergolic reactions; cyclopentadienyl compounds; fuming nitric acid

\section{Introduction}

In hypergolic reactions, two chemical reagents ignite spontaneously upon contact to liberate energy and gases in a non-explosive manner. Although hypergolic reactions find application in rocket fuels and propellants, their role in materials processing still remains largely unexplored. It is only lately that hypergolics has been introduced as a new synthesis tool in materials science, particularly for the fast and spontaneous preparation of carbon materials at ambient conditions in an energy liberating manner [1-5]. For this purpose, an organic compound serving as the carbon source and a strong oxidizer were spontaneously ignited upon contact at room temperature and atmospheric pressure to afford carbon without the need of any external source of heat (e.g., baking oven, hydrothermal treatment, or chemical vapor deposition). In this respect, hypergolic synthesis should be regarded as an energy-liberating rather than an energy-consuming process.

Typical paradigms developed from our group within this context include carbon nanosheets directly derived from self-ignitable lithium dialkylamides salts [1], highly crystalline graphite through 
spontaneous ignition of in situ derived acetylene-chlorine hypergolic mixture [2], carbon nanosheets or carbon dots from hypergolic mixtures based on nitrile rubber or Girard's reagent $\mathrm{T}$ and fuming nitric acid as a strong oxidizer [3], carbon nanosheets or fullerols from hypergolic pairs based on coffee grains or $\mathrm{C}_{60}$ and sodium peroxide as a strong oxidizer [4], and finally, carbon soot from the exothermic reaction between ferrocene and liquid bromine at ambient conditions [5]. In all instances, the reactions were immediate and exothermic at ambient conditions, with the released energy being further exploited in some cases for the production of useful work (chemical, thermoelectric, photovoltaic, etc.).

A classic guide to organic compounds that are hypergolic on contact with strong oxidizers is Bretherick's Handbook of Reactive Chemical Hazards. By scrutinizing the vast number of chemicals listed there, we came across the cyclopentadiene-fuming nitric acid hypergolic pair (e.g., cyclopentadiene ignites upon contact with concentrated nitric acid [6]). Motivated by this information, we set out to study the carbon residue produced from the hypergolic reaction of cyclopentadienyllithium with fuming nitric acid, the former acting as the carbon source. It turned out that the produced carbon was amorphous and oxidized, mostly adopting discoidal morphology. Interestingly, ferrocene and cobaltocene bearing cyclopentadienyl rings in their structure also reacted hypergolicly with fuming nitric acid, resulting instead in magnetic nanopowders as the major phase $\left(\gamma-\mathrm{Fe}_{2} \mathrm{O}_{3}\right.$, metallic $\mathrm{Co}$ ). Therefore, cyclopentadienyl compounds, due to a large variety, are potentially interesting starting reagents for the hypergolic synthesis of a wider range of materials at ambient conditions in an energy-liberating manner (i.e., exothermically). In a broader sense, hypergolics offer a new synthesis scheme in materials science that is complementary to other established preparative methods, such as solid state, sol-gel, wet chemistry, precipitation, hydrothermal, pyrolysis, flame spray pyrolysis, chemical vapor deposition, etc.

At its current form, the present method serves as a proof of concept in materials science. Certain advantages of hypergolic reactions include the fast rate, spontaneity, and exothermic character at ambient conditions. In addition, hypergolics under the materials science perspective provides a useful way to deal with waste from rocket propellants. On the other side, although such reactions are pretty safe to run at a small scale in the lab, nevertheless, safety issues should be seriously taken into consideration upon scaling up the method (e.g., pilot set-ups closely resembling rocket fuel engines should be rather devised). In a parallelism relation, flame-spray pyrolysis is another example of hazardous technique involving hot flames and flammable hydrocarbons; however, the method is now widely used in labs and industries for the large-scale production of nanomaterials. This was only possible due to systematic technical upgrades over time towards the advancement of safe and sophisticated apparatus. In another point, the reaction yields (typically 2-15\%), though acceptable, are lower than those of classic preparative methods, such as precipitation, sol-gel, or flame-spray pyrolysis. But this mainly has to do with the carbon or inorganic content of the precursors used in the hypergolic reactions. The study or discovery of new hypergolic pairs that will provide even higher yields remains a challenge.

\section{Materials and Methods}

Synthesis was conducted in a fume hood with ceramic tile bench. A glass test tube (diameter: $1.5 \mathrm{~cm}$; length: $15 \mathrm{~cm})$ was charged with $0.5 \mathrm{~g}$ cyclopentadienyllithium $\left(97 \% \mathrm{C}_{5} \mathrm{H}_{5} \mathrm{Li}\right.$, Sigma-Aldrich, St. Louis, MO, USA) followed by the slow, dropwise addition of $0.5 \mathrm{~mL}$ fuming nitric acid $(100 \%$, Sigma-Aldrich, St. Louis, MO, USA). The cyclopentadienyl salt and fuming nitric acid ignited instantly upon contact to from a carbon residue within the test tube. The residue was collected and washed thoroughly with de-ionized water $\left(\approx 5.5 \times 10^{-5} \mathrm{~S} \mathrm{~m}^{-1}\right)$, acetone $(\geq 99 \%$ Merck KGaA, Darmstadt, Germany), and tetrahydrofuran (THF, $\geq 99 \%$ Merck KGaA, Darmstadt, Germany) prior to drying at $80^{\circ} \mathrm{C}$. A fine carbon powder was obtained at a yield of $2 \%$, compared to the weight of the cyclopentadienyl compound used for reaction $\left(\mathrm{N}_{2}\right.$ specific surface area: $\left.225 \mathrm{~m}^{2} \mathrm{~g}^{-1}\right)$. The whole process is illustrated in Figure 1. 


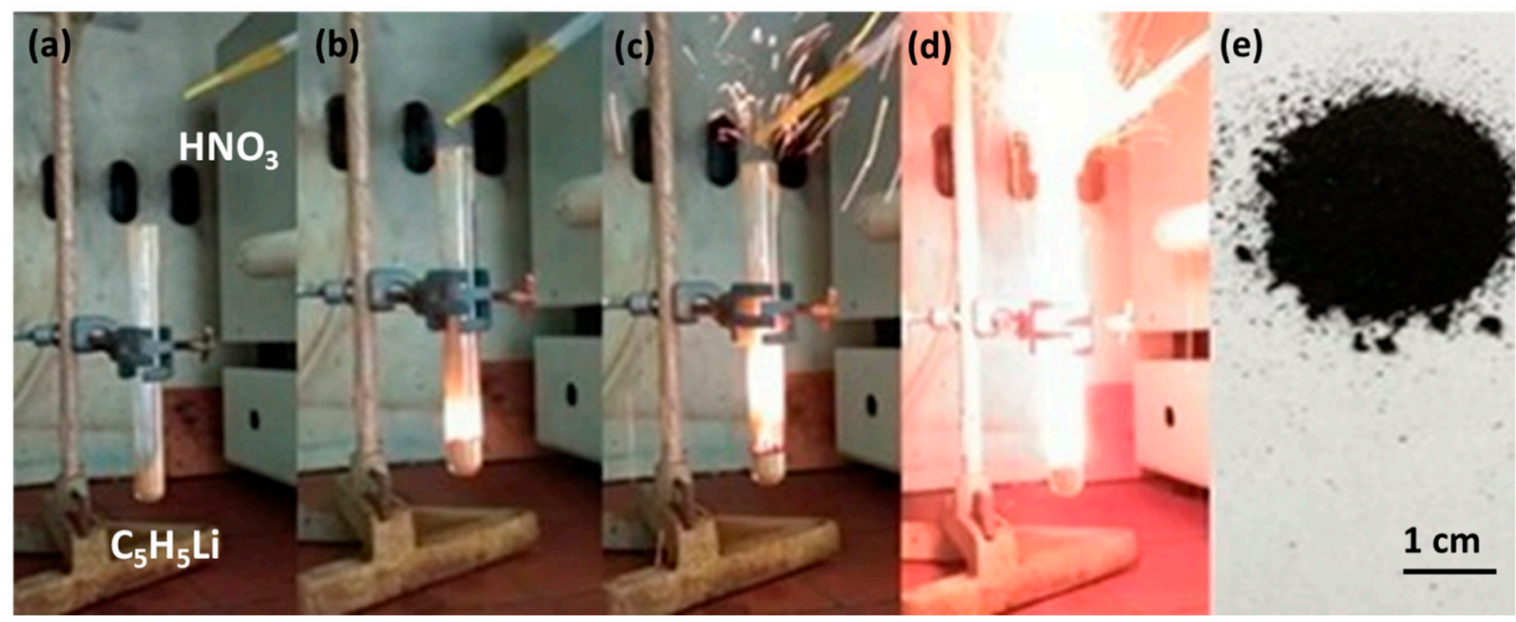

Figure 1. The hypergolic reaction of cyclopentadienyllithium with fuming nitric acid produced a carbon fine powder: (a) The test tube contained the salt before nitric acid addition with a pipette. (b-d) Dropwise addition of the acid triggered ignition, giving off an intense burst of flame. (e) The residue within the tube was collected and washed to give a fine black powder.

Similarly to the cyclopentadienyllithium salt, ferrocene ( $\geq 98 \%$, Sigma-Aldrich, St. Louis, MO, USA) and cobaltocene ( $98 \%$, Sigma-Aldrich, St. Louis, MO, USA) also reacted hypergolicly with fuming nitric acid as above to afford magnetic nanopowders, such as $\gamma-\mathrm{Fe}_{2} \mathrm{O}_{3}$ and metallic $\mathrm{Co}$, at a yield of $10 \%$ compared to the weights of the cyclopentadienyl compounds used for reaction $\left(\mathrm{N}_{2}\right.$ specific surface areas: 36 for $\gamma-\mathrm{Fe}_{2} \mathrm{O}_{3}$ and $28 \mathrm{~m}^{2} \mathrm{~g}^{-1}$ for $\mathrm{Co}$ ). Characteristically, the ignition of ferrocene by fuming nitric acid is illustrated in Figure 2. Due to a small yield, reactions were repeated several times in order to collect enough material for characterizations. In addition, all presented reactions were completed within less than a minute, thus allowing a rapid product formation at ambient conditions. Lastly, there was a sudden release of a sizeable amount of energy upon direct contact of the reagents at ambient conditions (i.e., energy-releasing process). The heat produced from the reactions provided the necessary energy needed for the carbonization of the cyclopentadienyl compounds (e.g., in situ pyrolysis).

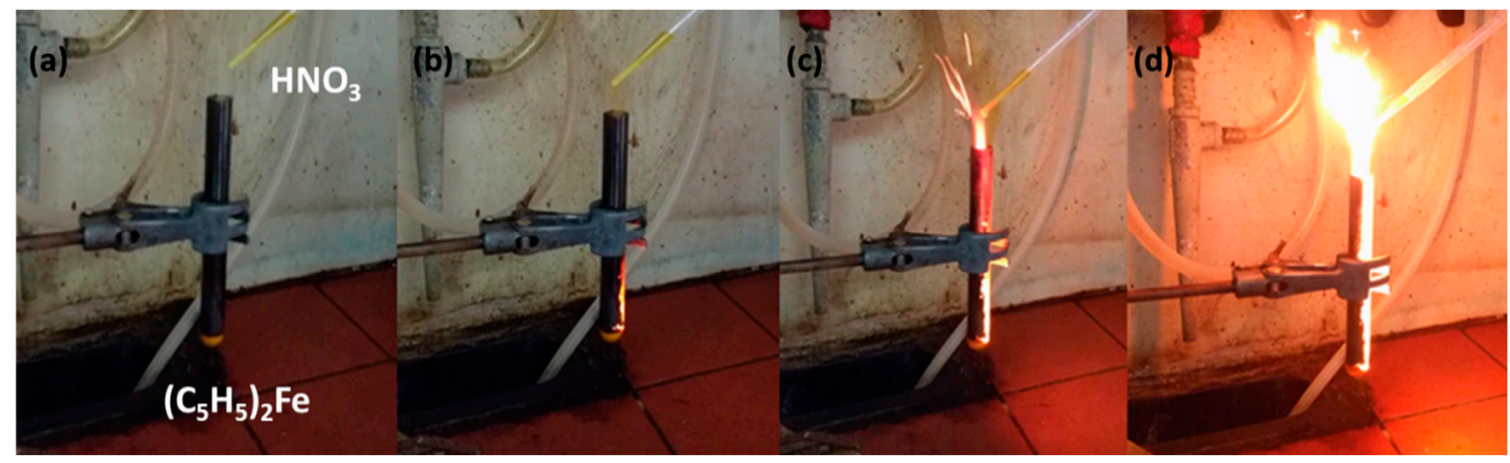

Figure 2. (a-d) Dropwise addition of fuming nitric acid into a test tube containing ferrocene powder resulted in the hypergolic ignition of the cyclopentadienyl compound by the acid (e.g., intense burst of flame) and the subsequent formation of $\gamma-\mathrm{Fe}_{2} \mathrm{O}_{3}$ inside the tube. The magnetic solid was recovered by scratching off and washing the dark brown residue from inside the tube.

$\mathrm{X}$-ray diffraction $(\mathrm{XRD})$ was conducted on background-free $\mathrm{Si}$ wafers using $\mathrm{Cu} \mathrm{K} \alpha$ radiation from a Bruker Advance D8 diffractometer (Bruker, Billerica, MA, USA). Attenuated total reflection infrared spectroscopy (ATR-IR) measurements were performed using a Jasco IRT-5000 microscope coupled with a FT/IR-4100 spectrometer (Jasco, Easton, MD, USA). The ZnSe prism of the ATR objective had a $250 \mu \mathrm{m}$ area in contact with the sample. The background was subtracted and the baseline was corrected for all spectra. Raman spectra were obtained on a DXR Raman microscope (Thermo Scientific Waltham, 
MA, USA) using a laser excitation line at $455 \mathrm{~nm}, 2 \mathrm{~mW}$. X-ray photoelectron spectroscopy (XPS) was performed on a PHI VersaProbe II (Physical Electronics, Chanhassen, MN, USA) spectrometer using Al $\mathrm{K} \alpha$ source $(15 \mathrm{kV}, 50 \mathrm{~W})$. Deconvolution of spectra was made using the MultiPak software package (Ulvac-PHI, Inc., Miami, FL, USA). The $\mathrm{N}_{2}$ adsorption-desorption isotherms were measured at $77 \mathrm{~K}$ on a volumetric gas adsorption analyzer (3Flex, Micromeritics, Norcross, GA, USA). The carbon, $\gamma-\mathrm{Fe}_{2} \mathrm{O}_{3}$ and Co samples were outgassed at $130{ }^{\circ} \mathrm{C}$ for $12 \mathrm{~h}$ under high vacuum $\left(10^{-4} \mathrm{~Pa}\right)$ before measurements. Specific surface areas were determined with the Brunauer-Emmett-Teller (BET) method for all three samples. Atomic force microscopy (AFM) images were obtained in the tapping mode with a Bruker Multimode 3D Nanoscope (Ted Pella Inc., Redding, CA, USA) using a microfabricated silicon cantilever type TAP-300G, with a tip radius of $<10 \mathrm{~nm}$ and a force constant of approximately 20-75 $\mathrm{N} \mathrm{m}^{-1}$ The transmission electron microscopy (TEM) study was performed using the instrument JEM HR-2100, (JEOL Ltd., Tokyo, Japan) operated at $200 \mathrm{kV}$ in bright-field mode. SEM images were obtained using a JEOL JSM-6510 LV SEM Microscope (Ltd., Tokyo, Japan) equipped with an X-Act EDS-detector by Oxford Instruments (Abingdon, Oxfordshire, UK, an acceleration voltage of $20 \mathrm{kV}$ was applied). Magnetic measurements were carried out at room temperature using a Vibrating Sample Magnetometer (VSM) Lakeshore model 7300 (Westerville, OH USA).

\section{Results and Discussion}

\subsection{Cyclopentadienyllithium- $\mathrm{HNO}_{3}$ hypergolic Pair}

The carbon obtained from the cyclopentadienyllithium- $\mathrm{HNO}_{3}$ hypergolic pair was easily identified by the XRD and Raman techniques. The XRD pattern of the product exhibited a very broad reflection centered at $\mathrm{d}_{002}=4.0 \AA$ (Figure 3, top), signaling the formation of highly disordered carbon [7]. In this case, the lack of periodicity between layers could be ascribed to the rapid release of gases during reaction that pushes the carbon layers apart from each other (e.g., exfoliation). At the same time, Raman spectroscopy (Figure 3, bottom) gave characteristic D (1354) and G (1582 $\mathrm{cm}^{-1}$ ) bands with intensity ratio $\left(\mathrm{I}_{\mathrm{D}} / \mathrm{I}_{\mathrm{G}} \sim 1\right)$ typical of amorphous carbon containing both $\mathrm{sp}^{3} / \mathrm{sp}^{2}$ domains [7].
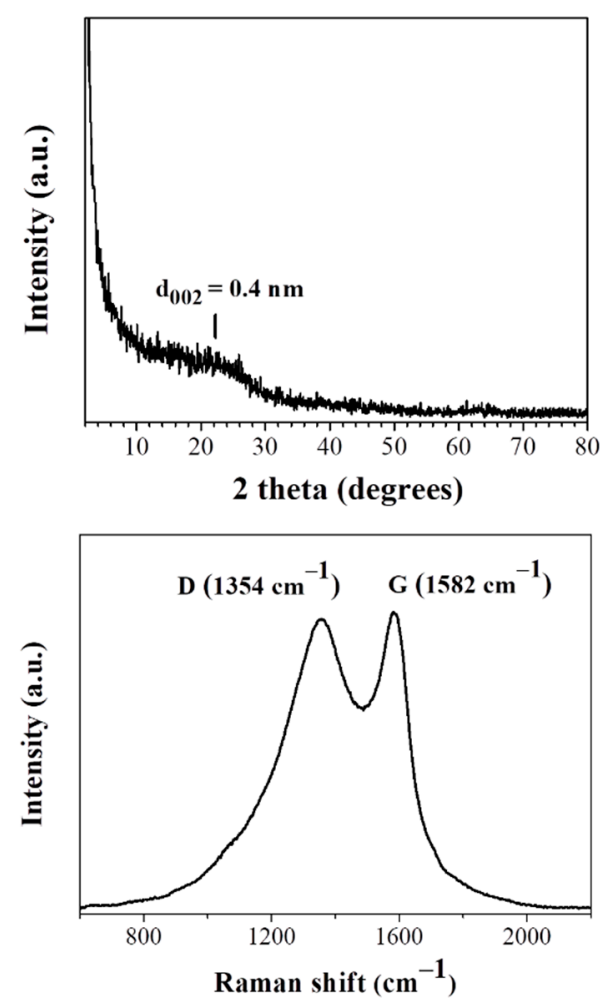

Figure 3. XRD pattern (top) and Raman spectrum (bottom) of the carbon sample. 
The ATR infrared spectrum of the carbon solid displayed several characteristic absorptions bands (Figure 4). The broad band at $1200 \mathrm{~cm}^{-1}$ was due to $\mathrm{C}-\mathrm{O} / \mathrm{C}-\mathrm{OH}$ stretching, the band at $1580 \mathrm{~cm}^{-1}$ was ascribed to the presence of $\mathrm{C}=\mathrm{C} / \mathrm{C}=\mathrm{N}$ bonds, the weak shoulder at $1700 \mathrm{~cm}^{-1}$ was assigned to carbonyl groups $\mathrm{C}=\mathrm{O}$, and finally, the weak peaks near $3000 \mathrm{~cm}^{-1}$ to residual $\mathrm{C}-\mathrm{H}$ moieties. The presence of oxygen-containing groups pinpointed oxidation of the carbon surface, in line with the XPS results discussed in the next paragraph. Furthermore, the IR-active $C=C$ stretching vibration was quite close to the Raman-active $G$ band of the solid as a result of symmetry breaking by lattice defects, nitrogen doping, and/or surface oxidation (e.g., the so-called "doing Raman with IR") [8]. Lastly, residual hydrogens normally resulted from the organic salt precursor by ignition.

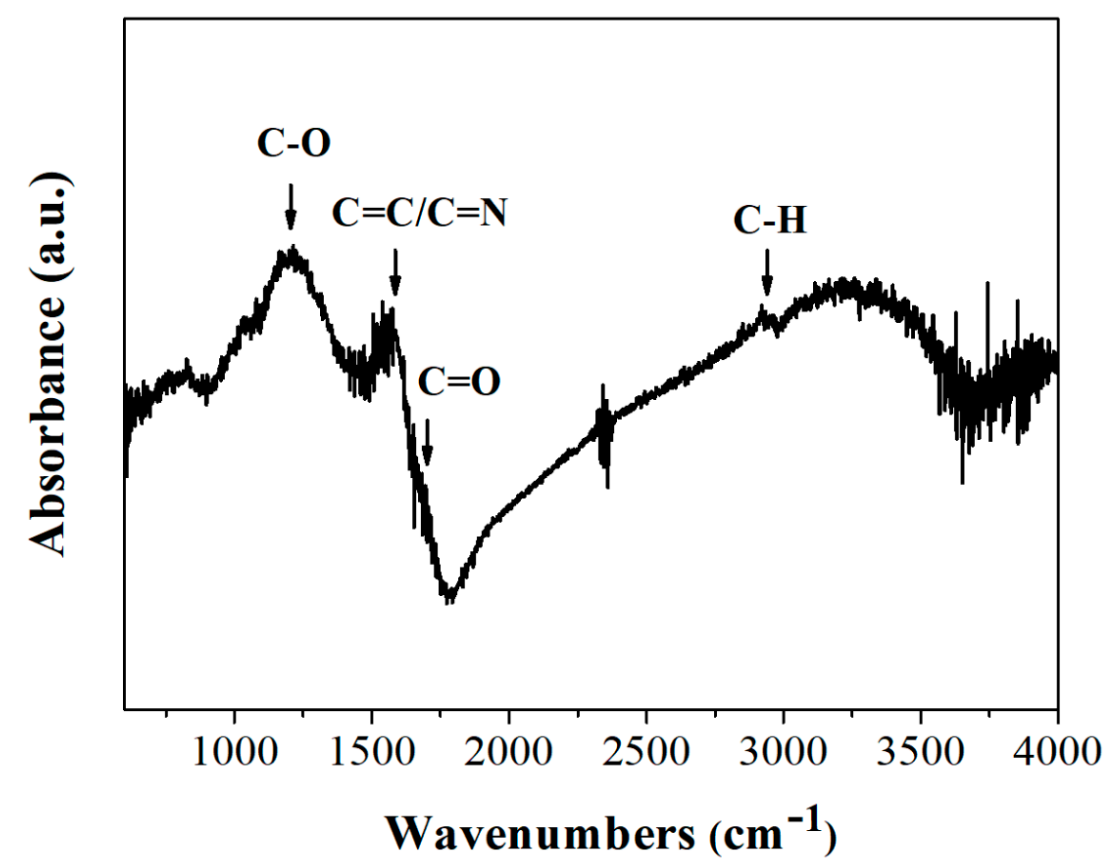

Figure 4. ATR infrared spectrum of the carbon solid.

The XPS survey spectrum of the sample showed predominantly carbon ( $81 \%)$ and, to a lesser extent, oxygen (16.1\%) and nitrogen (2.9\%) (Figure $5 \mathrm{a})$. Note that no trace of lithium was detected in the region between $55-57 \mathrm{eV}$, which is characteristic of the element. Oxygen was due to ignition in open air (e.g., air-oxidation), while nitrogen due to the nitric acid used for reaction (e.g., $\mathrm{N}$-doping by $\mathrm{HNO}_{3}$ ) [9]. According to this reference, nitric acid served as the nitrogen source through the release of reactive nitrogen species that bind to the carbon surface by the aim of heat produced from the exothermic reaction. Deconvolution of the high-resolution $\mathrm{C} 1 \mathrm{~s}$ spectrum (Figure $5 \mathrm{~b}$ ) revealed different carbon bonding states $(C=C, C-C, C-O / C-N, C=O$, and satellite) $[10,11]$. Therefore, the sample consisted of $\mathrm{sp}^{2}$ and $\mathrm{sp}^{3}$ carbon configurations with an intense presence of $\mathrm{C}-\mathrm{O}$ (which overlapped with the $\mathrm{C}-\mathrm{N}$ bond region) and $\mathrm{C}=\mathrm{O}$ bonds, in agreement with the atomic composition of the sample. The presence of oxygen-containing groups, which may act as surface binding sites, and the relatively good specific surface area of the sample are attractive structural features in carbon-based adsorption/removal technologies (e.g., carbon filters).

An AFM study of the sample revealed two types of morphologies, namely carbon nanosheets and discoidal nanocarbon [12] (Figure 6a-c). The micron-sized thin nanosheets appeared with an average thickness of ca. $2.5 \mathrm{~nm}$ according to section analysis (Figure 6a). On the other hand, the submicron-sized discoidal carbon exhibited a bimodal thickness distribution with thicknesses of ca. 1 (Figure 6b) and ca. $5 \mathrm{~nm}$ (Figure 6c) (e.g., the presence of thinner and thicker discs). The discoidal particles appeared flat as evidenced by their 3D morphology (Figure 6d). The appearance frequency of the discoidal morphology in the sample was up to $92 \%$ (Figure 6e), thereby largely dominating 
the sum. It is worth noting that nearly $77 \%$ of the discs appeared to have similar thickness with graphene $(1 \mathrm{~nm})$. Hence, we can safely conclude that the good specific surface area of the sample stems in part from the highly exfoliated state and nanosized thickness of the discs. The disc-like morphology of the sample was additionally confirmed by SEM and TEM microscopies, showing both individual and aggregated nanodiscs of submicron lateral dimensions (Figure 7).
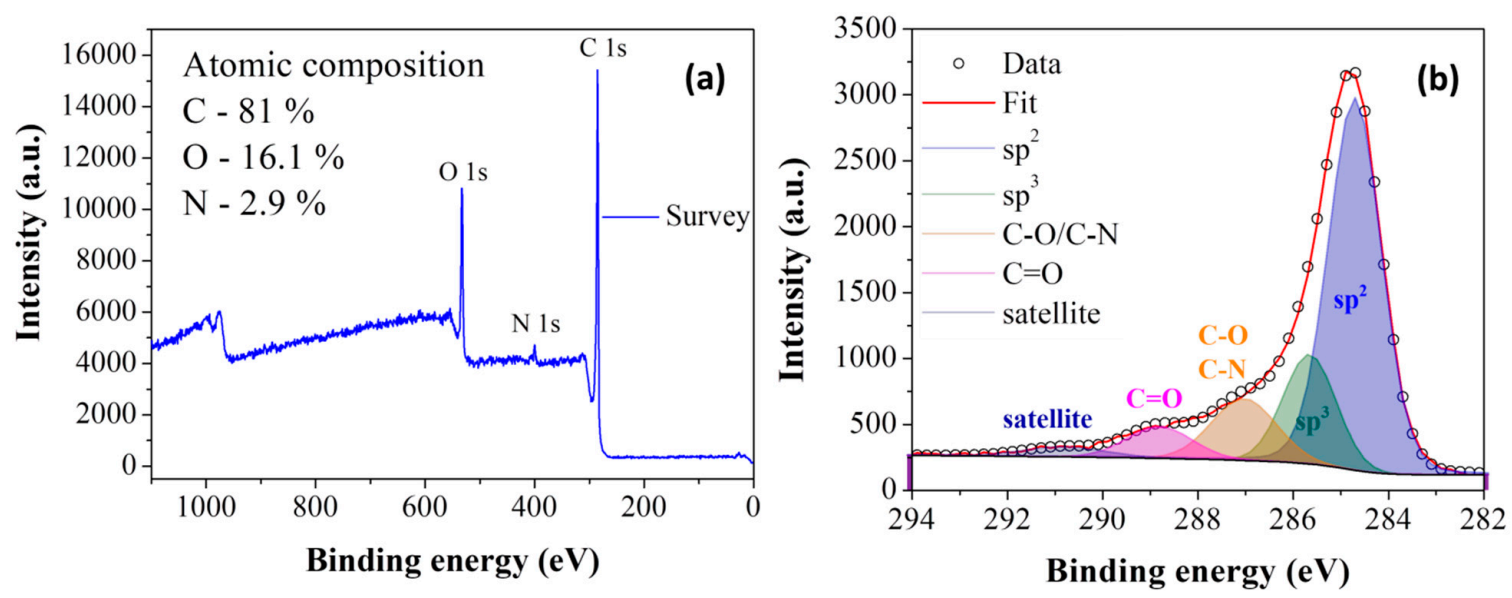

Figure 5. (a) XPS survey spectrum of the carbon sample with the respective atomic composition. (b) High-resolution XPS C1s envelope with assigned deconvoluted components.

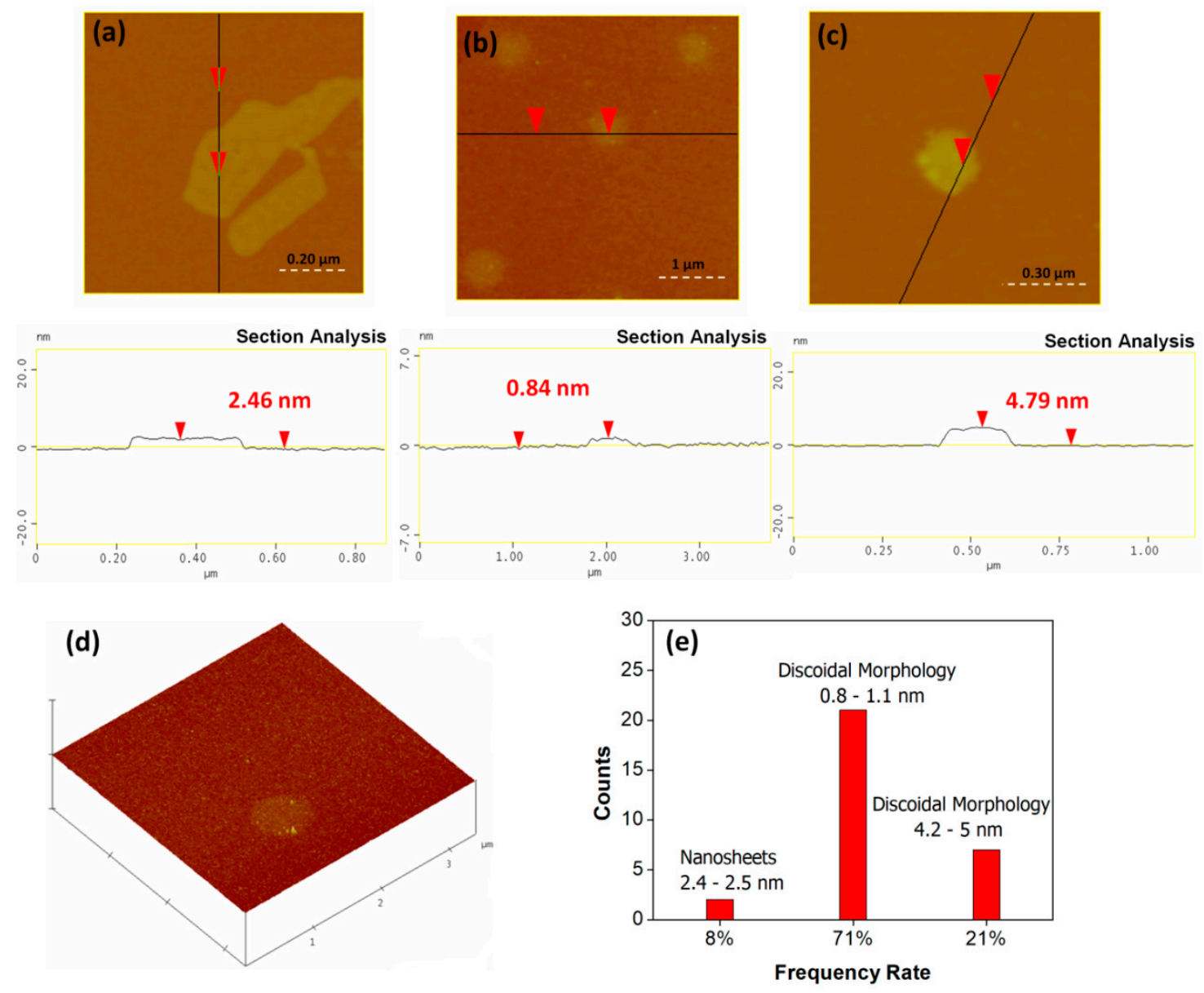

Figure 6. (a-c) AFM images of cross-sectional analysis of the carbon sample (nanosheets and discs). (d) 3D morphology of a flat disc. (e) Histogram of frequency rate of the different morphologies along with their thicknesses. 


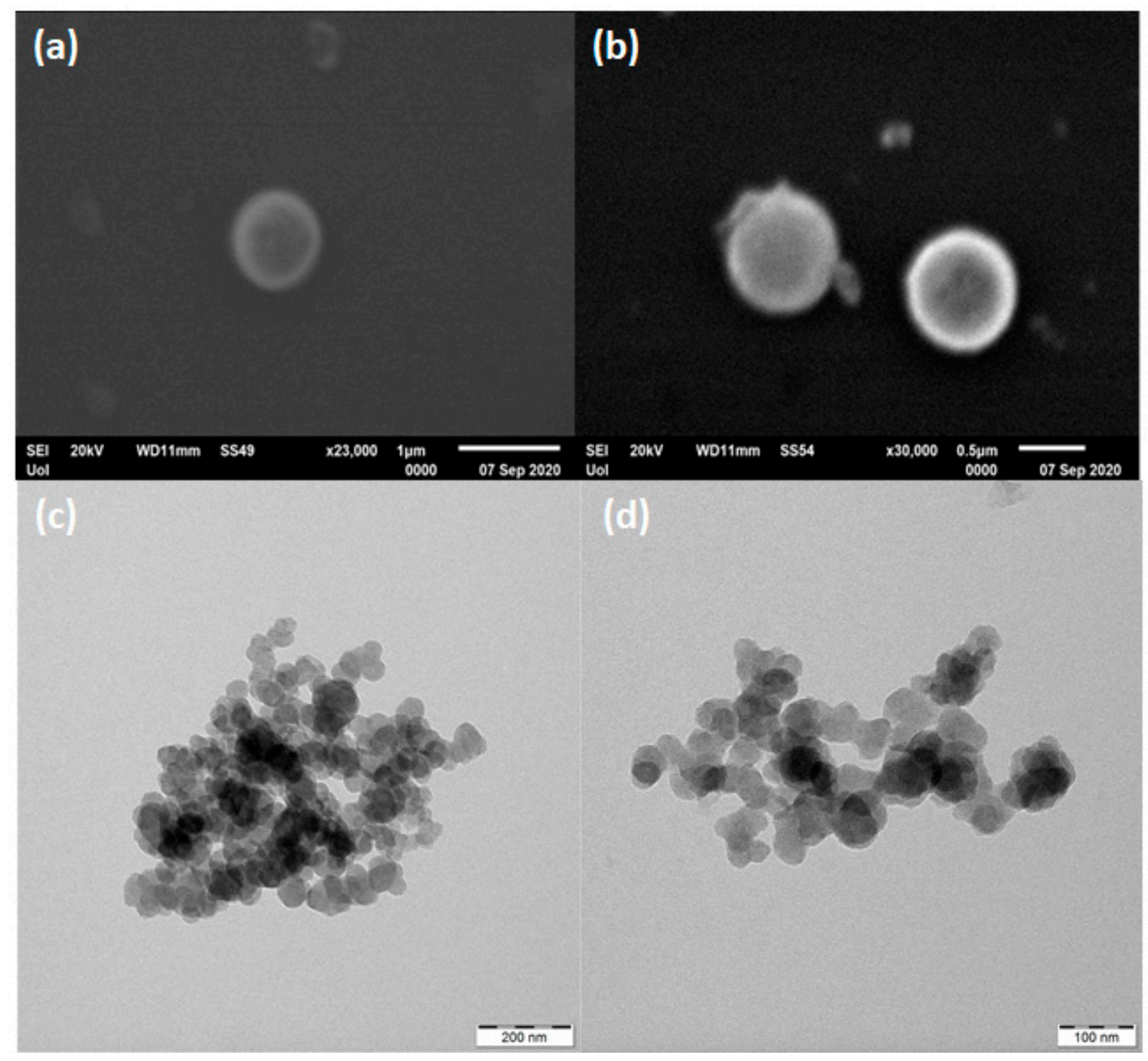

Figure 7. SEM $(\mathbf{a}, \mathbf{b})$ and TEM $(\mathbf{c}, \mathbf{d})$ images of carbon discs.

\subsection{Ferrocene- and Cobaltocene- $\mathrm{HNO}_{3}$ Hypergolic Pairs}

Another interesting class of cyclopentadienyl compounds are metallocenes, such as ferrocene and cobaltocene. In the present study, the pure compounds reacted hypergolicly on contact with fuming nitric acid to give magnetic $\gamma-\mathrm{Fe}_{2} \mathrm{O}_{3}$ and $\mathrm{Co}$ nanoparticles as the major phase. Figure 8 displays the indexed XRD patterns of the ferrocene-derived $\gamma-\mathrm{Fe}_{2} \mathrm{O}_{3}$ and cobaltocene-derived Co magnetic phases. Even though magnetite and $\gamma-\mathrm{Fe}_{2} \mathrm{O}_{3}$ have similar XRD patterns, nevertheless, $\mathrm{Fe}_{3} \mathrm{O}_{4}$ formation requires anaerobic conditions in order to avoid air-oxidation into $\gamma-\mathrm{Fe}_{2} \mathrm{O}_{3}$. In this respect, ignition in air under strongly oxidizing conditions rather favors the formation of the more stable $\gamma-\mathrm{Fe}_{2} \mathrm{O}_{3}$ phase. Furthermore, some residual metallic $\mathrm{Fe} / \mathrm{Fe}_{3} \mathrm{C}$ phases were also detected due to possible carbothermal reduction of the oxide. Turning to the cobalt case, traces of cobalt oxides were additionally observed as a result of metal oxidation. Generally, ferrocene is a well-known precursor to magnetic iron oxides [13,14]; similarly, cobaltocene is a well-known precursor to metallic cobalt [15]. 

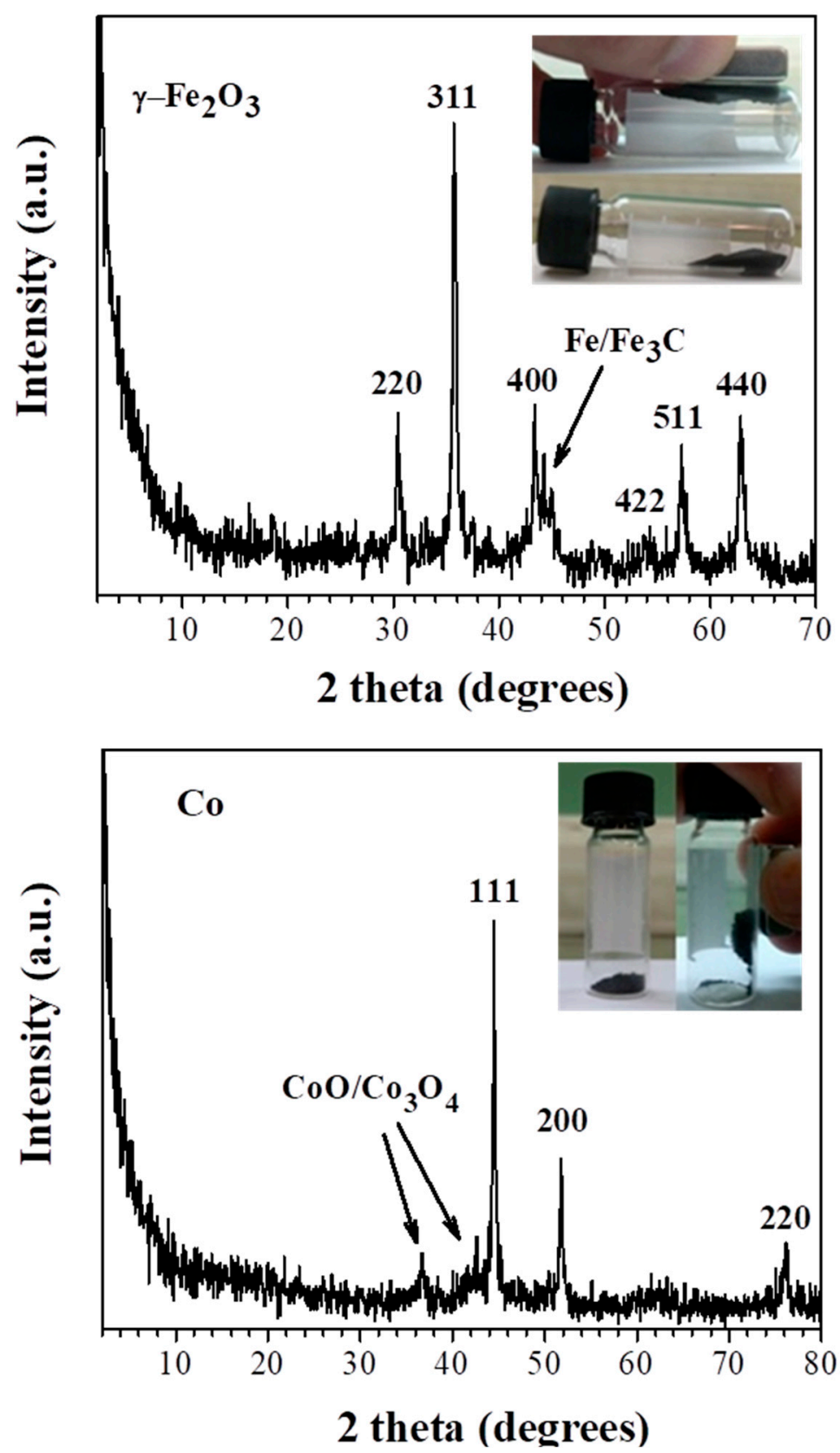

Figure 8. XRD patterns of (top) the as-derived $\gamma-\mathrm{Fe}_{2} \mathrm{O}_{3}$ and (bottom) Co magnetic powders. The insets show the strong attraction of the magnetic solids by $\mathrm{Nd}$-magnet.

The transition metals in ferrocene and cobaltocene should normally catalyze carbon combustion during ignition by $\mathrm{HNO}_{3}$; however, the samples did contain residual carbon. Indeed, thermal gravimetric analysis in the air showed clear weight losses of 30-35\% due to combustion of amorphous carbon near $300{ }^{\circ} \mathrm{C}$. Practically, this means that mild heat treatment of the samples between $300-400{ }^{\circ} \mathrm{C}$ in the air should effectively remove most of the amorphous carbon without severely affecting the structure of the magnetic phases (e.g., $\gamma-\mathrm{Fe}_{2} \mathrm{O}_{3}$ is stable up to $400{ }^{\circ} \mathrm{C}$, whereas Co oxidation in air commences $>400^{\circ} \mathrm{C}$ ). The AFM study of the magnetic solids revealed the presence of spherical nanoparticles with an average size of 8-11 nm for $\gamma-\mathrm{Fe}_{2} \mathrm{O}_{3}$ and 19-21 nm for metallic Co (Figure 9a,b). These values were in a good agreement with those calculated from XRD and the Scherrer equation. The round shape of the nanoparticles was additionally confirmed by the corresponding 3D morphology images of the nanoparticles (Figure 9c,d). 

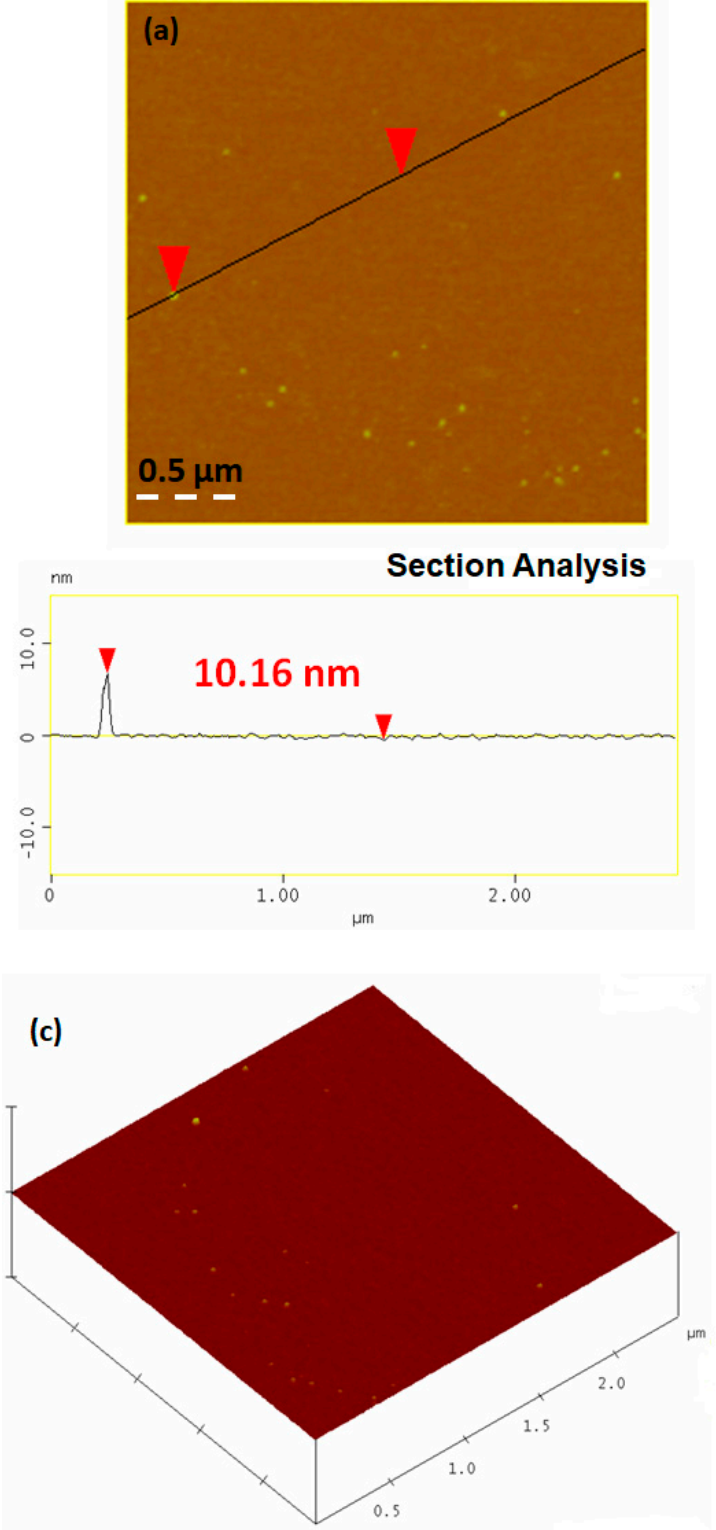

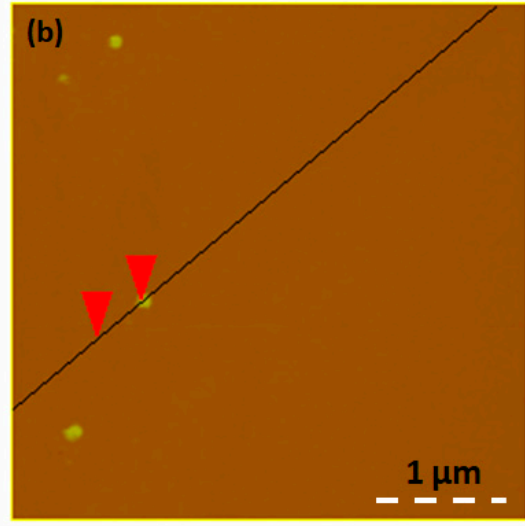

Section Analysis

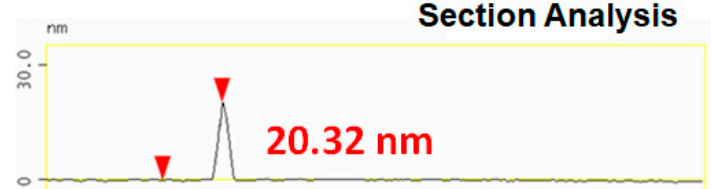

(d)

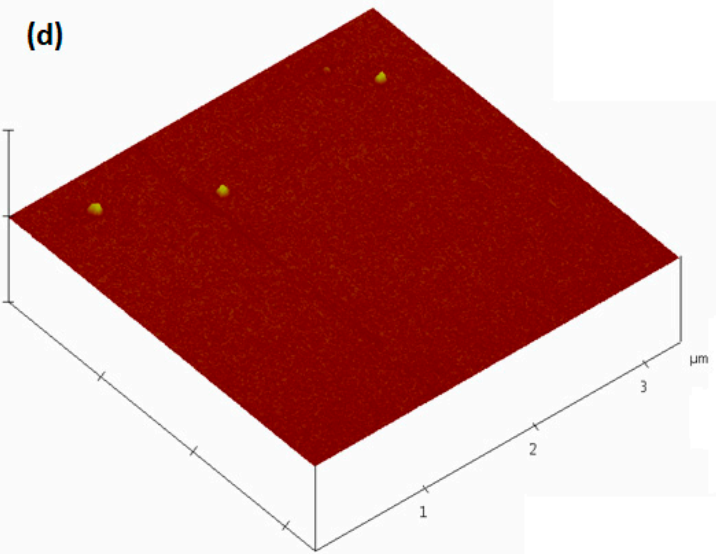

Figure 9. Cross-sectional analysis $(\mathbf{a}, \mathbf{b})$ and $3 \mathrm{D}$ morphology $(\mathbf{c}, \mathbf{d})$ of the magnetic nanoparticles. $(\mathbf{a}, \mathbf{c}) \gamma-\mathrm{Fe}_{2} \mathrm{O}_{3}$ and $(\mathbf{b}, \mathbf{d}) \mathrm{Co}$.

The corresponding magnetic powders were strongly attracted by the Nd-magnet (insets, Figure 8). Figure 10 shows the magnetization curves of the ferrocene- and cobaltocene-derived magnetic solids at room temperature. The nanoparticles did not exhibit hysteresis (e.g., almost negligible coercivity). On the other hand, the saturation magnetization values were found to be $71 \mathrm{emu} \mathrm{g}{ }^{-1}$ for $\gamma-\mathrm{Fe}_{2} \mathrm{O}_{3}$ and $70 \mathrm{emu} \mathrm{g}^{-1}$ for $\mathrm{Co}$. These values were lower than those of the bulk phases $\left(\gamma-\mathrm{Fe}_{2} \mathrm{O}_{3}: 83 \mathrm{emu} \mathrm{g}^{-1}\right.$; $\mathrm{Co}$ : $162 \mathrm{emu} \mathrm{g}^{-1}$ ) due to the quantum size effect. Overall, the magnetization data confirmed the nanosized dimensions of the obtained particles.

Likewise, cobaltocene, nickelocene (99\% Acros Organics ${ }^{\mathrm{TM}}$, Morris County, NJ, USA) afforded magnetic nickel nanoparticles after ignition with fuming nitric acid. Moreover, the method was useful for the synthesis of alloy nanoparticles as well. For this purpose, equimolar amounts of cobaltocene and nickelocene were ground in the presence of acetone in a mortar and pestle to obtain a homogenous powder after drying. Acetone is a good solvent for both metallocenes, thus allowing molecular blending of the precursors. Following, the mixture was ignited by fuming nitric acid to afford $\mathrm{CoNi}$ magnetic nanoparticles displaying the characteristic (111), (200), and (220) reflections of the alloy [16]. 
Lastly, chromocene (95\% Sigma-Aldrich, St. Louis, MO, USA) reacted similarly as above, to give instead ceramic $\mathrm{Cr}_{2} \mathrm{O}_{3}$. The corresponding XRD patterns of all solids are given in Figure 11. Therefore, depending on the cyclopentadienyl precursor many more materials can be foreseen and explored through hypergolics in the near future.
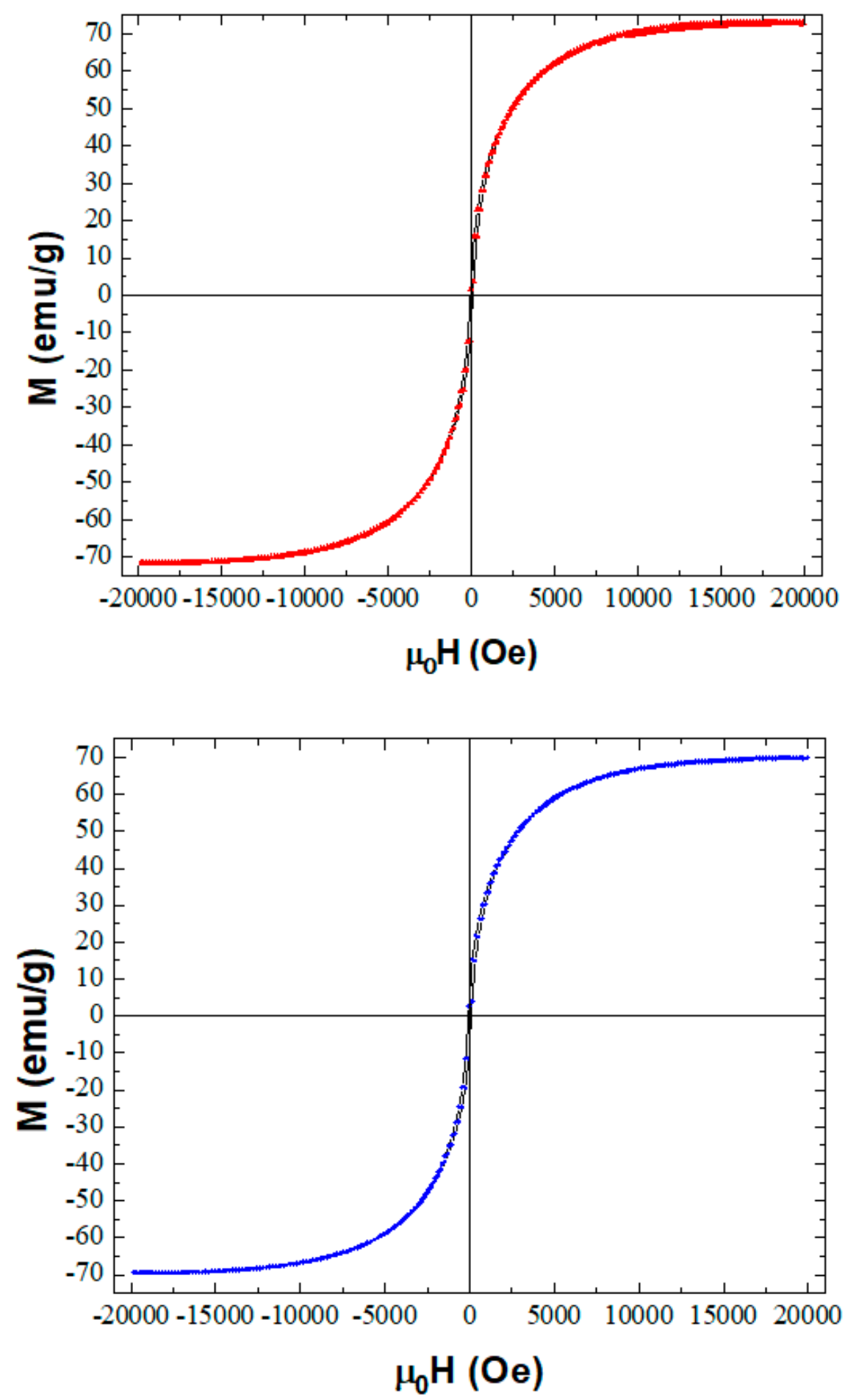

Figure 10. Magnetization curves of the ferrocene-(top red loop) and cobaltocene-derived (bottom blue loop) magnetic solids at room temperature. 


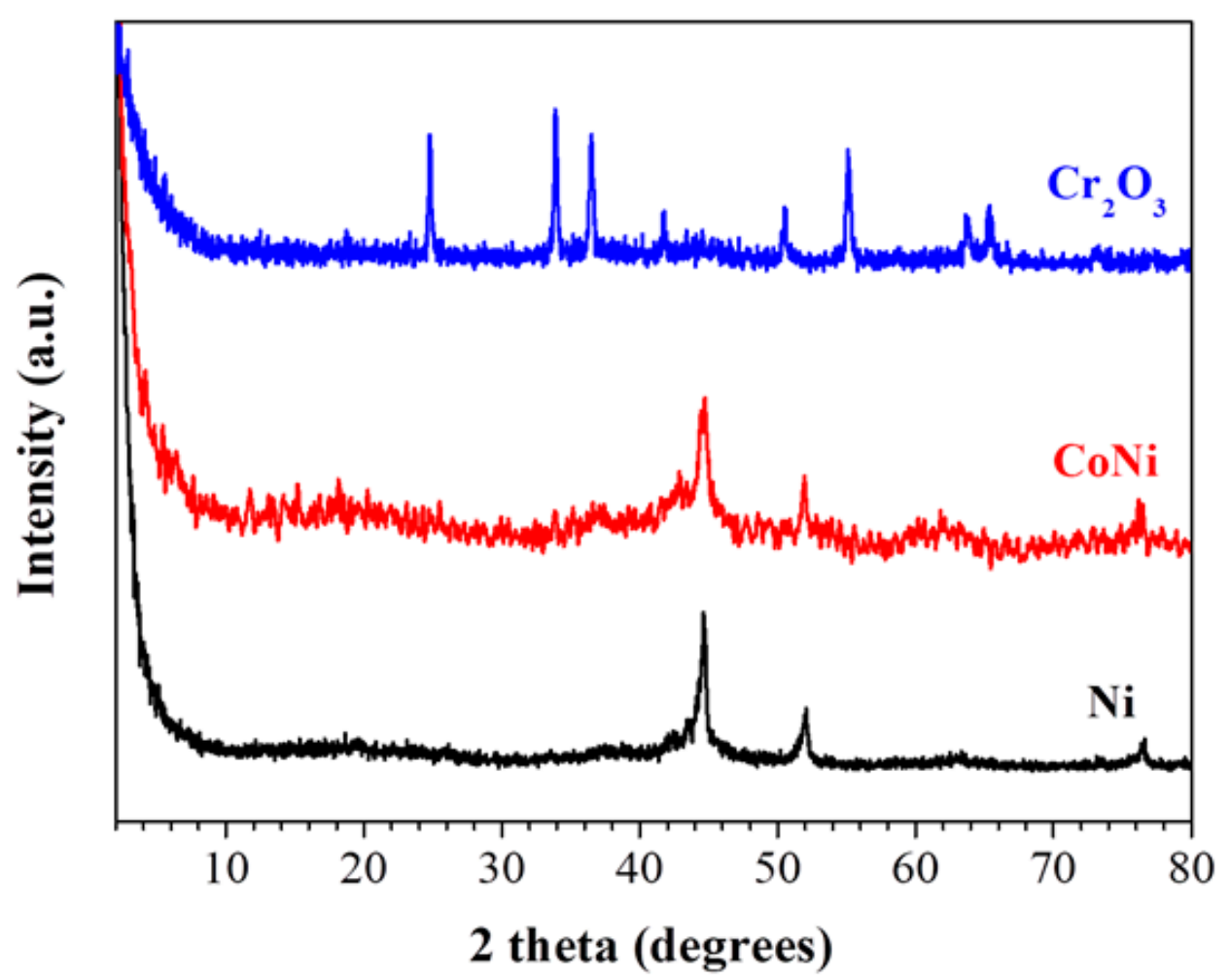

Figure 11. XRD patterns of the as-derived $\mathrm{Ni}$, CoNi alloy, and $\mathrm{Cr}_{2} \mathrm{O}_{3}$ nanoparticles.

\section{Conclusions}

Cyclopentadienyl compounds are versatile reagents in the hypergolic synthesis of different types of nanomaterials, such as carbon and inorganic. Indicatively, the hypergolic reaction of cyclopentadienyllithium with fuming nitric acid gave a carbon residue mostly composed of discoidal particles, whereas analogous reactions with ferrocene and cobaltocene afforded $\gamma-\mathrm{Fe}_{2} \mathrm{O}_{3}$ and Co magnetic nanoparticles, respectively. In all instances, the reactions were fast, spontaneous, and exothermic at ambient conditions, resulting in rapid product formation with fairly good yields. Based on these grounds, hypergolics merits further attention in materials science as a new preparative method of a broader class of nanomaterials.

Author Contributions: Conceptualization, experiments, and writing A.B.B. and D.G.; formal analysis, experiments, and writing N.C., V.Š., V.K., D.M., A.A., and M.A.K. All authors have read and agreed to the published version of the manuscript.

Funding: We acknowledge support of this work by the project "National Infrastructure in Nanotechnology, Advanced Materials and Micro-/Nanoelectronics" (MIS-5002772) which was implemented under the action "Reinforcement of the Research and Innovation Infrastructure", funded by the Operational Programme "Competitiveness, Entrepreneurship and Innovation" (NSRF 2014-2020), and co-financed by Greece and the European Union (European Regional Development Fund). This research was also co-financed by Greece and the European Union (European Social Fund- ESF) through the Operational Programme "Human Resources Development, Education and Lifelong Learning" in the context of the project "Strengthening Human Resources Research Potential via Doctorate Research" (MIS-5000432), implemented by the State Scholarships Foundation (IKY). V.Š. acknowledges the assistance provided by the Research Infrastructure NanoEnviCz II from MEYS CZ (LM2018124) and the support from the Internal Student Grant Agency of the Palacký University in Olomouc, Czech Republic (IGA_PrF_2020_022). V.Š. and V.K. also acknowledge the financial support from MEYS CZ under the project CZ.02.1.01/0.0/0.0/16_019/0000754.

Conflicts of Interest: The authors declare no conflict of interest. 


\section{References}

1. Baikousi, M.; Chalmpes, N.; Spyrou, K.; Bourlinos, A.B.; Avgeropoulos, A.; Gournis, D.; Karakassides, M.A. Direct production of carbon nanosheets by self-ignition of pyrophoric lithium dialkylamides in air. Mater. Lett. 2019, 254, 58-61. [CrossRef]

2. Chalmpes, N.; Spyrou, K.; Bourlinos, A.B.; Moschovas, D.; Avgeropoulos, A.; Karakassides, M.A.; Gournis, D. Synthesis of highly crystalline graphite from spontaneous ignition of in situ derived acetylene and chlorine at ambient conditions. Molecules 2020, 25, 297. [CrossRef] [PubMed]

3. Chalmpes, N.; Asimakopoulos, G.; Spyrou, K.; Vasilopoulos, K.C.; Bourlinos, A.B.; Moschovas, D.; Avgeropoulos, A.; Karakassides, M.A.; Gournis, D. Functional carbon materials derived through hypergolic reactions at ambient conditions. Nanomaterials 2020, 10, 566. [CrossRef] [PubMed]

4. Chalmpes, N.; Spyrou, K.; Vasilopoulos, K.C.; Bourlinos, A.B.; Moschovas, D.; Avgeropoulos, A.; Gioti, C.; Karakassides, M.A.; Gournis, D. Hypergolics in carbon nanomaterials synthesis: New paradigms and perspectives. Molecules 2020, 25, 2207. [CrossRef] [PubMed]

5. Chalmpes, N.; Tantis, I.; Bakandritsos, A.; Bourlinos, A.B.; Karakassides, M.A.; Gournis, D. Rapid carbon formation from spontaneous reaction of ferrocene and liquid bromine at ambient conditions. Nanomaterials 2020, 10, 1564. [CrossRef] [PubMed]

6. Trent, C.H.; Zucrow, M.J. The hypergolic reaction of dicyclopentadiene with white fuming nitric acid. J. Am. Rocket Soc. 1951, 21, 129-131. [CrossRef]

7. Roh, J.-S. Structural study of the activated carbon fiber using laser Raman spectroscopy. Carbon Lett. 2008, 9 , 127-130. [CrossRef]

8. Ferrari, A.C.; Rodil, S.E.; Robertson, J. Interpretation of infrared and Raman spectra of amorphous carbon nitrides. Phys. Rev. B 2003, 67, 155306. [CrossRef]

9. D'Arsié, L.; Esconjauregui, S.; Weatherup, R.S.; Wu, X.; Arter, W.E.; Sugime, H.; Cepek, C.; Robertson, J. Stable, efficient p-type doping of graphene by nitric acid. RSC Adv. 2016, 6, 113185-113192. [CrossRef]

10. Bakandritsos, A.; Pykal, M.; Błoński, P.; Jakubec, P.; Chronopoulos, D.D.; Poláková, K.; Georgakilas, V.; Čépe, K.; Tomanec, O.; Ranc, V.; et al. Cyanographene and graphene acid: Emerging derivatives enabling high-yield and selective functionalization of graphene. ACS Nano 2017, 11, 2982-2991. [CrossRef] [PubMed]

11. Zoppellaro, G.; Bakandritsos, A.; Tuček, J.; Błoński, P.; Susi, T.; Lazar, P.; Bad'ura, Z.; Steklý, T.; Opletalová, A.; Otyepka, M.; et al. Microwave energy drives "on-off-on" spin-switch behavior in nitrogen-doped graphene. Adv. Mater. 2019, 31, 1902587. [CrossRef] [PubMed]

12. Zygouri, P.; Tsoufis, T.; Kouloumpis, A.; Patila, M.; Potsi, G.; Sevastos, A.A.; Sideratou, Z.; Katsaros, F.; Charalambopoulou, G.; Stamatis, H.; et al. Synthesis, characterization and assessment of hydrophilic oxidized carbon nanodiscs in bio-related applications. RSC Adv. 2018, 8, 122-131. [CrossRef]

13. Amara, D.; Margel, S. Solventless thermal decomposition of ferrocene as a new approach for the synthesis of porous superparamagnetic and ferromagnetic composite microspheres of narrow size distribution. J. Mater. Chem. 2011, 21, 15764-15772. [CrossRef]

14. Amara, D.; Margel, S. Synthesis and characterization of elemental iron and iron oxide nano/microcomposite particles by thermal decomposition of ferrocene. Nanotechnol. Rev. 2013, 2, 333. [CrossRef]

15. Dormans, G.J.M.; Meekes, G.J.B.M.; Staring, E.G.J. OMCVD of cobalt and cobalt silicide. J. Cryst. Growth 1991, 114, 364-372. [CrossRef]

16. Wei, X.-W.; Zhou, X.-M.; Wu, K.-L.; Chen, Y. 3-D flower-like NiCo alloy nano/microstructures grown by a surfactant-assisted solvothermal process. CrystEngComm 2011, 13, 1328-1332. [CrossRef]

(C) 2020 by the authors. Licensee MDPI, Basel, Switzerland. This article is an open access article distributed under the terms and conditions of the Creative Commons Attribution (CC BY) license (http://creativecommons.org/licenses/by/4.0/). 